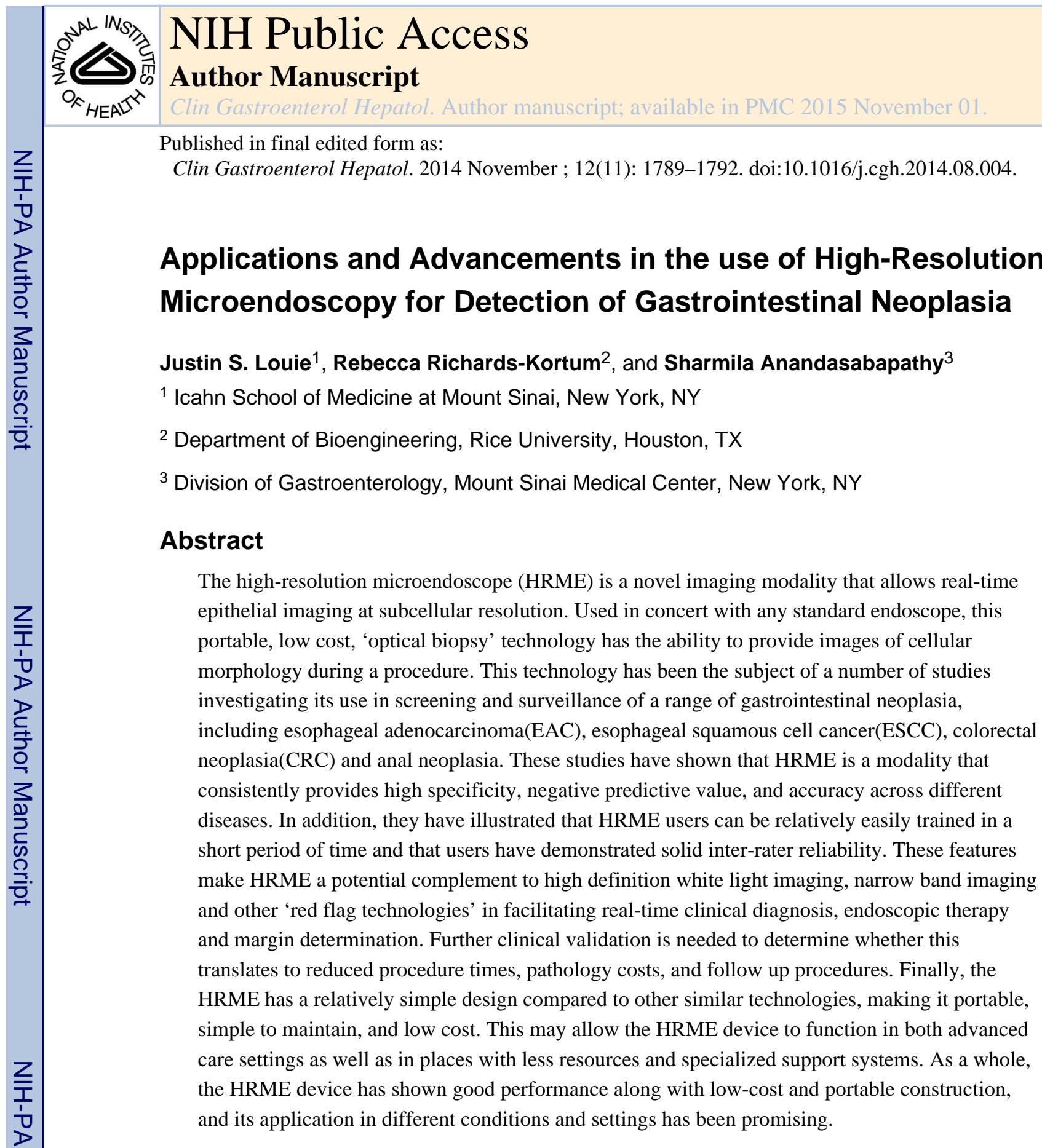

The high-resolution microendoscope (HRME) is a novel imaging modality that allows real-time epithelial imaging at subcellular resolution. Used in concert with any standard endoscope, this portable, low cost, 'optical biopsy' technology has the ability to provide images of cellular morphology during a procedure. This technology has been the subject of a number of studies investigating its use in screening and surveillance of a range of gastrointestinal neoplasia, including esophageal adenocarcinoma(EAC), esophageal squamous cell cancer(ESCC), colorectal neoplasia(CRC) and anal neoplasia. These studies have shown that HRME is a modality that consistently provides high specificity, negative predictive value, and accuracy across different diseases. In addition, they have illustrated that HRME users can be relatively easily trained in a short period of time and that users have demonstrated solid inter-rater reliability. These features make HRME a potential complement to high definition white light imaging, narrow band imaging and other 'red flag technologies' in facilitating real-time clinical diagnosis, endoscopic therapy and margin determination. Further clinical validation is needed to determine whether this translates to reduced procedure times, pathology costs, and follow up procedures. Finally, the HRME has a relatively simple design compared to other similar technologies, making it portable, simple to maintain, and low cost. This may allow the HRME device to function in both advanced care settings as well as in places with less resources and specialized support systems. As a whole, the HRME device has shown good performance along with low-cost and portable construction, and its application in different conditions and settings has been promising.

(C) 2014 The AGA Institute All rights reserved.

Corresponding Author: Sharmila Anandasabapathy, MD Baylor Global Initiatives, Cullen Building, One Baylor Plaza, Houston, TX 77030 Sharmila.Anandasabapathy@mountsinai.org.

Publisher's Disclaimer: This is a PDF file of an unedited manuscript that has been accepted for publication. As a service to our customers we are providing this early version of the manuscript. The manuscript will undergo copyediting, typesetting, and review of the resulting proof before it is published in its final citable form. Please note that during the production process errors may be discovered which could affect the content, and all legal disclaimers that apply to the journal pertain.

Potential competing interests: Rebecca Richards-Kortum serves as an unpaid scientific advisor to Remicalm LLC, holds patents related to optical diagnostic technologies that have been licensed to Remicalm LLC, and holds minority ownership in Remicalm LLC. The remaining authors declare no conflict of interest. 


\section{Technological primer}

The high-resolution microendoscope(HRME) is a novel imaging modality, first described by Muldoon et al to present the system's construction and demonstrate its sub-cellular resolution imaging capabilities. ${ }^{1}$ The HRME consists of three components: a thin-flexible fiber-optic probe, a combined light source and camera, and a laptop or tablet based processor. ${ }^{2}$ It requires topical application of fluorescent contrast agents, such as proflavine, typically used at $0.01 \%$ concentration. The $3-\mathrm{m}$ long probe has 30,000 individual optical fibers that total $1 \mathrm{~mm}$ in diameter; this probe is inserted through the accessory channel of a standard endoscope (similar to an endocytoscope or probe-based confocal microendoscope). Images are recorded by placing the distal tip of the fiber bundle into direct contact with the mucosa after application of the contrast agent, with each optical fiber acting as an individual pixel of the image. Illumination is provided by a battery powered $455 \mathrm{~nm}$ blue light-emitting diode(LED) transmitted through the imaging probe to the mucosal surface. The LED illumination excites the fluorophore, with the emitted light collected by the bundle and directed into a digital camera connected to the computer. This allows real-time mucosal imaging at subcellular resolution $(4.4 \mu \mathrm{m}), 1000 \mathrm{x}$ magnification and a frame rate of $12 \mathrm{fps}$. The field of view of the HRME depends on the diameter of the active area of the fiber bundle, usually $720 \mu \mathrm{m}$. The probe can be disinfected and reused approximately 60-75 times, before the probe tip requires polishing. The advantages of this imaging technology include its portability, ease of use, and low cost, with current prototypes costing $<\$ 3500$ in components.

\section{Findings}

The first description of this device in gastrointestinal neoplasia was associated with surveillance of Barrett's Esophagus(BE). ${ }^{3}$ Since that time, there have been multiple studies exploring its efficacy as an imaging modality in esophageal adenocarcinoma(EAC), esophageal squamous cell cancer(ESCC), colorectal neoplasia(CRC), and anal cancer.(Table 1) There have also been ex vivo studies investigating its possible use in hepatobiliary neoplasia and gastric adenocarcinoma, which will not be addressed here.

\section{Esophageal Neoplasia:}

One of the earliest studies investigated the accuracy and inter-rater reliability of HRME for diagnosing Barrett's neoplasia. ${ }^{4}$ In this trial, 20 gastroenterologists with no microendoscopic experience and three expert ( $>50$ cases) microendoscopists completed training and subsequently interpreted images in a test set comprised of HRME images taken from 28 consecutive patients undergoing surveillance for BE. Overall sensitivity for identification of high-grade dysplasia or cancer was $90 \%$, with $82 \%$ specificity, $94 \%$ negative predictive value(NPV), compared to histology, and moderate inter-rater reliability $(\kappa=0.56)$. Interestingly, no significant differences in accuracy were observed between experts and novices.

In a current prospective in vivo trial of patients with $\mathrm{BE}$, standard high-definition white-light endoscopy(WLE) is being compared to WLE+HRME, with subjects undergoing both targeted and random 'optical' biopsies with HRME. ${ }^{5}$ Preliminary results for 49 patients 
suggest a superior sensitivity (100\% vs. $50 \%)$ and comparable specificity ( $94.9 \%$ vs. $96.3 \%$ ) in the combined WLE+HRME group compared to WLE alone. Furthermore, this study evaluated diagnostic yield, with HRME (37.5\%) outperforming WLE alone (1.71\%), and suggesting that HRME could negate the need for $92.2 \%$ of individual biopsies, with $70.8 \%$ of patients not requiring any biopsies at all.

Several studies have explored the applicability of HRME in ESCC. A large prospective international multicenter trial comparing Lugol's chromoendoscopy(LCE) to LCE guided HRME for the detection of ESCC was recently performed and the preliminary results presented at the 2014 American Gastroenterological Association meetings. ${ }^{6}$ In this study, 147 consecutive patients undergoing endoscopic screening or surveillance for esophageal squamous neoplasia were enrolled from four centers. Each patient was evaluated in real-time using LCE, followed by HRME evaluation of both Lugol's voiding areas and LCE normal areas. LCE guided HRME demonstrated significant improvement compared to LCE alone in specificity ( $88 \%$ vs. $48 \%$ ) and accuracy (90\% vs. $57 \%)$; with only a small but not statistically significant loss of sensitivity ( $90 \%$ vs. $97 \%$ ). These results translated into $34 \%$ of LCE positive biopsies potentially saved and an improved diagnostic yield from $14 \%$ to $45 \%$. Once again, there was no significant difference between novices and experts.

Automated image analysis has also been explored in ESCC. In an initial study, images taken from 177 patients were used to develop an algorithm, based on quantitative features, to identify high-grade dysplasia or ESCC. ${ }^{7}$ Optimal performance was obtained using mean nuclear area as the classification basis, resulting in high specificity (92-97\%), and NPV (98-99\%), when compared with histology.

\section{Colon and Anal Neoplasia:}

Another in vivo study explored the utility of HRME in differentiating colonic neoplasia. ${ }^{8}$ In this study, 171 polyps from 94 patients were identified on routine colonoscopy, imaged by HRME, and classified in real-time as neoplastic (adenomatous) or non-neoplastic. HRME had significantly higher accuracy (94\%), specificity (95\%), and positive predictive value $(87 \%)$ for the identification of neoplasia compared with WLE $(65 \%, 39 \%, 55 \%)$, without a loss of sensitivity or NPV. Furthermore, this trend persisted in sub-analysis of both small $(<10 \mathrm{~mm})$ and diminutive $(<5 \mathrm{~mm})$ polyps.

The role of HRME as an adjunct to high-resolution anoscopy was investigated in a study looking at detection of high-grade squamous intraepithelial lesions(HSIL). ${ }^{9}$ Forty one consecutive patients with abnormal anal cytology or prior HSIL underwent guided HRME imaging on Lugol's unstained areas, with subsequent biopsies. An image database was created and three expert HRME microendoscopists and 5 novice gastroenterologists and anoscopists reviewed a set of training images and then interpreted images in an independent test set. Overall, participants identified HSIL with high sensitivity (93\%), specificity (87\%), NPV (96\%), and accuracy (89\%), with slightly better specificity and accuracy in experts, and substantial inter-rater reliability for all groups $(\kappa=0.65)$.

This information was further employed to identify quantitative features that could increase the diagnostic utility of HRME during anoscopy. ${ }^{10} 62$ images were selected from the 
database and analyzed. Evaluation of eleven nuclear features yielded promising results, with a combination of three primary measurements yielding a sensitivity/specificity of $90 \%$ and $88 \%$.

\section{Importance}

These findings demonstrate that HRME is able to provide high accuracy for detecting neoplasia across a wide variety of gastrointestinal neoplasia. In particular, this modality has shown high specificity and NPV, supporting its predicted role in surveillance and diagnosis confirmation.

Furthermore, HRME images can be interpreted visually or using automated image analysis software .The quantitative imaging analysis algorithms presented demonstrate good accuracy in differentiating neoplasia and can be used to identify image features that are most diagnostically useful.

Additionally, studies indicate that endoscopists can be rapidly trained to interpret HRME images while maintaining high accuracy. In general, novice microendoscopists performed only slightly worse than experts in specificity and accuracy, with comparable sensitivity. This suggests that gastroenterologists who are new to HRME can be successfully and rapidly trained, in short periods of time, to use this technology with results similar to experts.

\section{Translation}

The ideal role for microendoscopy is not in screening, but in surveillance where it can enhance diagnostic accuracy and reduce the number of biopsies taken. With this in mind the high NPV of HRME is favorable for ruling out neoplasia. This could potentially improve overall cost effectiveness of endoscopic surveillance in conditions such as Barrett's or ulcerative colitis, as surveillance biopsies account for significant cost per procedure. Notably, if paired with a suitably sensitive widefield platform, such a combination would be able to rapidly survey large mucosal surfaces, identify suspicious areas, and subsequently interrogate those regions with high resolution.

Furthermore, HRME offers clinicians the ability to interrogate histologic features of suspicious lesions and evaluate post-resection margins in real-time during procedures. Such information can be used to make immediate clinical decisions at the point of care, potentially facilitating immediate minimally invasive therapies such as EMR, expediting margin determination during procedures, and reducing repeat procedures.

Additionally, the straightforward optical design of the device requires no scanning mirrors, complex light sources, or other moving parts, and the device as a whole is small and portable. This translates into a significantly decreased cost for production and maintenance of the device. This also decreases the need for specialized maintenance and may enable its use in both areas with high levels of support and those with less infrastructure or resources, such as in third world countries, where other optical imaging technologies would not be available and cost-saving is even more important.

Clin Gastroenterol Hepatol. Author manuscript; available in PMC 2015 November 01. 


\section{Roadblocks and/or limitations}

There are several current limitations on translating the HRME device into practice. Firstly, proflavine, the most frequently utilized contrast agent, is currently an investigational drug. While no comprehensive long-term studies of proflavine have been published, proflavinecontaining compounds, such as topical antiseptics, have been used extensively without reported mutagenic effects in humans.

An additional limitation is the probe's inability to image subsurface regions. The current HRME device yields images by placing the device tip into direct contact with tissue, limiting image acquisition to the superficial epithelium. However, the fiber-optic probe is small enough to pass through the lumen of a 16-gauge needle which could be used to penetrate into deeper layers of the epithelium, allowing imaging of these areas, though this has yet to be explored in clinical trials.

Another drawback is its limited field of view. Consequently, alone, HRME can only sample a small fraction of the mucosa at a time, presenting challenges, especially in heterogeneous regions of tissue. One solution lies in coupling the HRME with a widefield imaging system, using this system to identify suspicious areas, which are then inspected by HRME. Another recently developed technique is video mosaicing, where consecutive video frames are stitched together as the probe advances along tissue. ${ }^{11}$ This creates compiled images from areas much larger than a single field of view.

Relatedly, the use of the HRME device does add time to the length of procedures. Preliminary results in esophageal surveillance show that additional procedure length can range from 2 to 25 minutes depending on the size of the area of interest and the experience of the operator. ${ }^{5}$ Though this may limit its use in conditions with large areas to survey, this additional time may also be mitigated by coupling the HRME with a widefield system.

Finally, though the HRME device has demonstrated high accuracy and specificity, there have been few trials comparing it against other high-sensitivity imaging technologies such as confocal and narrow band imaging. Additional comparative studies, like the ESCC trial by Protano et al, will need to be done to accurately compare the performance and diagnostic yield of HRME to other optical imaging modalities. ${ }^{6}$

\section{Conclusions}

In conclusion, recent studies have demonstrated that HRME can effectively differentiate neoplastic from non-neoplastic tissue in a range of gastrointestinal tissue. High accuracy, specificity and NPV, make it an attractive complementary tool in the assessment of suspicious tissue, and combining HRME with an appropriate widefield platform has the potential to help endoscopists determine which lesions require biopsy, which can be removed and discarded, and which require endoscopic removal. Though it will never completely replace pathology, this new modality has the potential to reduce procedurerelated costs, not only by virtue of its low cost components, but also by decreasing procedure time, biopsy number, and return visits. Furthermore, such cost-effective, portable devices can potentially help fill gaps in settings where the infrastructure to support 
traditional endoscopy and pathology services are either not available or affordable. Further investigations are warranted to determine the ideal widefield technology to complement HRME, and directly compare it to other optical biopsy technologies.

\section{Acknowledgments}

Grant Information: National Cancer Institute/NIH RO1 CA140257, R21 CA156704-0 and R01 CA103830. The content is solely the responsibility of the authors and does not necessarily represent the official views of the National Cancer Institute or the National Institutes of Health."

\section{References}

1. Muldoon TJ, et al. Subcellular-resolution molecular imaging within living tissue by fiber microendoscopy. Opt Express. 2007; 15:16413-16423. [PubMed: 19550931]

2. Pierce M, et al. High-resolution Fiber-optic Microendoscopy for in situ Cellular Imaging. J Vis Exp. 2011; 47:2306. [PubMed: 21248707]

3. Muldoon TJ, et al. High-resolution imaging in Barrett's esophagus: a novel, low-cost endoscopic microscope. Gastrointest Endosc. 2008; 68(4):737-44. [PubMed: 18926182]

4. Vila PM, et al. Accuracy and interrater reliability for the diagnosis of Barrett's neoplasia among users of a novel, portable high-resolution microendoscope. Dis Esophagus. 2014; 27(1):55-62. [PubMed: 23442220]

5. Lee M, et al. Diagnostic Yield and Clinical Impact of a Low-Cost Microendoscope in the Early Diagnosis of Barrett's Associated Neoplasia: a Prospective, Single-Center Randomized Controlled Trial. DDW2014, Su2011.

6. Protano MA, et al. Accuracy of a High-Resolution, Low-Cost Microendoscope for the Early Detection of Esophageal Squamous Cell Neoplasia: a Prospective, International, Multicenter Trial. DDW2014, Mo1134.

7. Shin D, et al. Quantitative analysis of high-resolution microendoscopic images for diagnosis of esophageal squamous cell carcinoma. Accepted and in press, CGH.

8. Parikh ND, et al. In vivo diagnostic accuracy of high-resolution microendoscopy in differentiating neoplastic from non-neoplastic colorectal polyps: a prospective study. Am J Gastroenterol. 2014; 109(1):68-75. [PubMed: 24296752]

9. Varela B, et al. Mo1645 High-Resolution Microendoscopy(HRME) in the Detection of Anal Intraepithelial Neoplasia: Assessment of Accuracy and Interobserver Variability. Gastrointestinal Endoscopy. 2013; 77(5):AB456-AB457.

10. Varela B, et al. High-Resolution Microendoscopy(HRME) in the Detection of Anal Intraepithelial Neoplasia: a Quantitative Approach to Optimize Diagnostic Accuracy. DDW2014, Sa1168.

11. Bedard N, et al. Real-time video mosaicing with a high-resolution microendoscope. Biomed Opt Express. 2012; 3:2428-35. [PubMed: 23082285] 

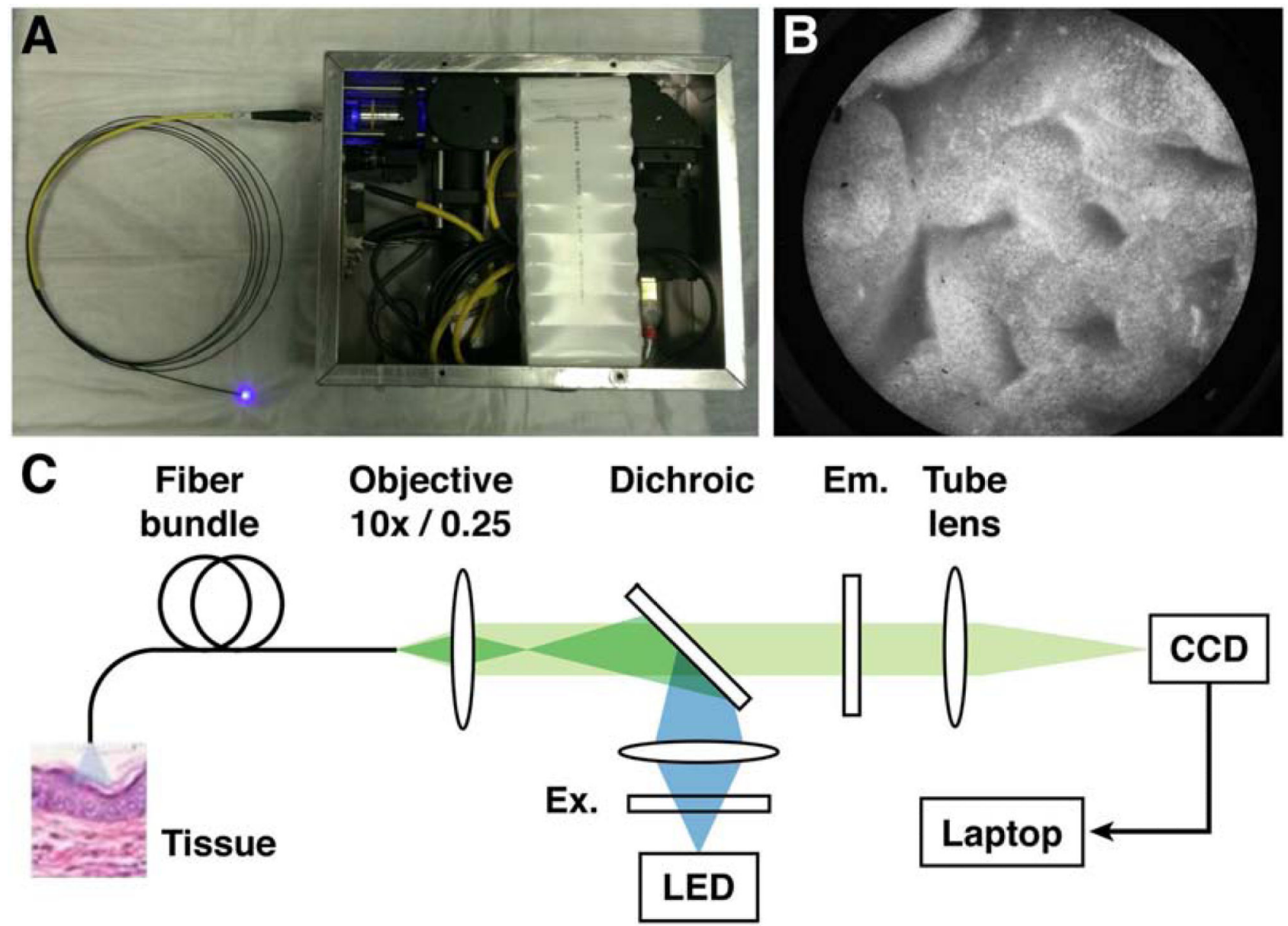

Figure 1.

(A) High Resolution Microendoscope device with probe. (B) HRME image of classic Barrett's Esophagus. (C) Schematic diagram: CCD, charge-coupled device/camera; Em, emission filter; Ex, excitation filter; LED, light-emitting diode. 
Table 1

HRME Performance Characteristics

\begin{tabular}{|l|l|l|l|l|l|l|}
\hline & Study & Sensitivity & Specificity & PPV & NPV & Accuracy \\
\hline EAC & Vila (2014) & 0.90 & 0.82 & 0.72 & 0.94 & $0.90 / 0.84^{*}$ \\
\hline & Lee (2014) & 1 & 0.949 & 0.372 & 1 & 0.95 \\
\hline ESCC & Protano (2014) & $0.90 / 0.90$ & $0.89 / 0.88$ & $0.55 / 0.40$ & $0.97 / 0.98$ & $0.90 / 0.89$ \\
\hline & Shin & $0.84-0.93$ & $0.92-0.97$ & $0.67-0.81$ & $0.98-0.99$ & 0.94 \\
\hline Colon & Parikh (2014) & 0.94 & 0.95 & 0.87 & 0.91 & 0.94 \\
\hline Anal & Varela (2013) & $0.87 / 0.97$ & $0.92 / 0.84$ & $0.85 / 0.77$ & $0.93 / 0.98$ & $0.90 / 0.89$ \\
\hline & Varela (2014) & 0.90 & 0.88 & & & \\
\hline
\end{tabular}

All results in this chart are given for per-biopsy analyses

* (Expert/Novice) 